\title{
Determination of Activated Slip Systems in Experimentally Deformed Olivine- Orthopyroxene Polycrystals using EBSD
}

\author{
René de Kloe*, Martyn Drury** and J.K. Farrer*** \\ * EDAX BV, PO Box 4144, 5004 JC Tilburg, The Netherlands \\ ** Vening Meinesz Research School of Geodynamics, Faculty of Earth Sciences, Utrecht \\ University, PO Box 80021, 3508 TA Utrecht, The Netherlands \\ *** TSL 392 East 12300 South, Draper, Utah, USA
}

Dislocation microstructures were studied in two sets of experimentally deformed partially molten olivine-orthopyroxene rocks containing 0.8-1.3\% melt. One set was deformed by Hitchings et al. ${ }^{1}$ and showed a strong creep strength dependence on orthopyroxene content (fig. 1a). The second set of experiments was carried out in 1991 by M.R. Drury in order to reproduce these results (De Kloe ${ }^{2}$ ). Within this latter sample set no correlation between orthopyroxene content and creep strength was found (fig. 1b). The observed variation in creep strength between the samples pointed to differences in activated dislocation slip systems. The creep strength of the stronger samples indicated that the strongest olivine slip system, (010)[001]-slip, was rate controlling. The creep strength of the weaker materials suggested that the weakest olivine slip system, (010)[100]-slip was rate-controlling, with lattice mismatches being accommodated by melt-enhanced grain boundary processes.

TEM weak beam dark field imaging was applied to verify the activation of these slip systems in selected samples. Dislocations and dislocation arrays or subgrain walls typical to either slip system were found in all samples and individual grains could contain dislocations that belonged to only one of the slip systems. No slip system was dominant in any sample. Because of the limited sample volume that could be studied in the TEM, electron backscatter diffraction analysis in the SEM was used to investigate the abundance of the dislocation slip systems that produced the subgrain boundaries in olivine. EBSD measurements allowed determination of the rotation axes that describe the misorientation accommodated by subgrain boundaries, although the measurement error in the determination of rotation axes for subgrain boundaries with misorientations smaller than $5^{\circ}$ can be as large as $30^{\circ}$. Confident identification of the crystallography of the dislocations forming the subgrain boundaries was possible because the number of possible slip systems in olivine is limited (fig. 2). EBSD measurements (fig. 3, 4) showed that subgrain boundaries formed by the weak (010)[100] slip system were more abundant in the stronger material, while the majority of the subgrain rotation axes in the weaker material indicated slip along an intermediate strength slip system, $(0 \mathrm{kl})[100]$. Additionally it was found that within larger olivine grains, clusters of subgrain boundaries occurred with similar rotation axes. Because such clusters exhibited different rotation axes, it is concluded that different dislocation slip systems dominated in different parts of the grain. These observations indicate that the observed correlation between the experimentally observed creep strength and the creep strength of olivine deforming by specific dislocation slip systems is coincidental.

It is concluded: (1) that EBSD analysis can be confidently used to identify dislocation slip systems that produce subgrain boundaries in olivine crystals, (2) that the observed strength variations between the sample sets were not related to the activation of different dislocation slip systems, and (3) that specific dislocation slip systems may be dominantly activated in specific volumes inside crystals and not homogeneously throughout the grains. 


\section{References:}

[1] R.S. Hitchings et al., Phys. Earth Planet. Inter. 55, 277, (1989)

[2] R. De Kloe, Ph.D.-thesis, Geologica Ultraiectina 201, Utrecht University, 173, (2001)

[3] This work was part of the PIONIER preient "Deformation mechanisms, rheology and tectonics of the Earth's upper mantle", funded by $\mathrm{NWO}$ project No. 030-75-346

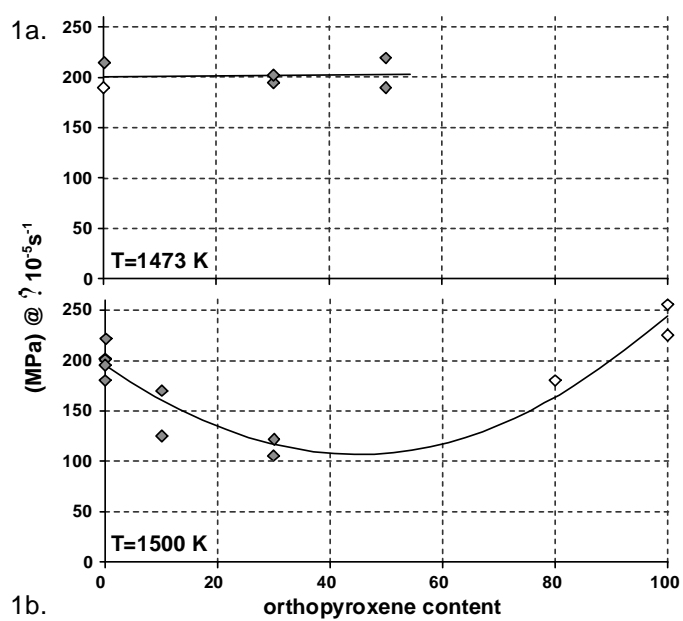

FIG. 1 a) Experimental data from M.R. Drury $\left(\right.$ De $\left.\mathrm{Kloe}^{2}\right)$ b) Experimental data from Hitchings et al. ${ }^{1}$.
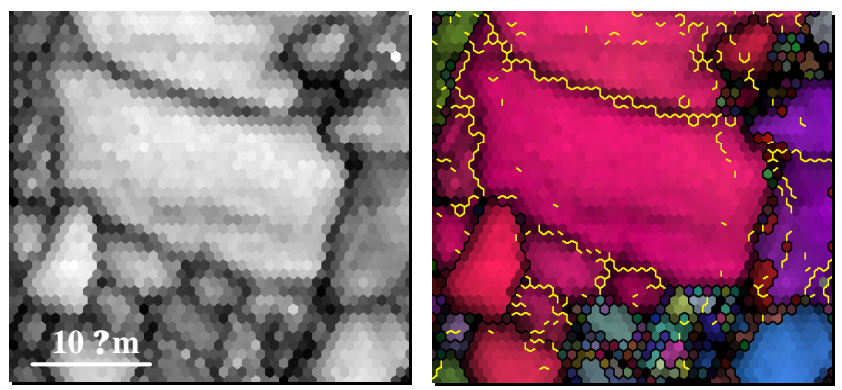

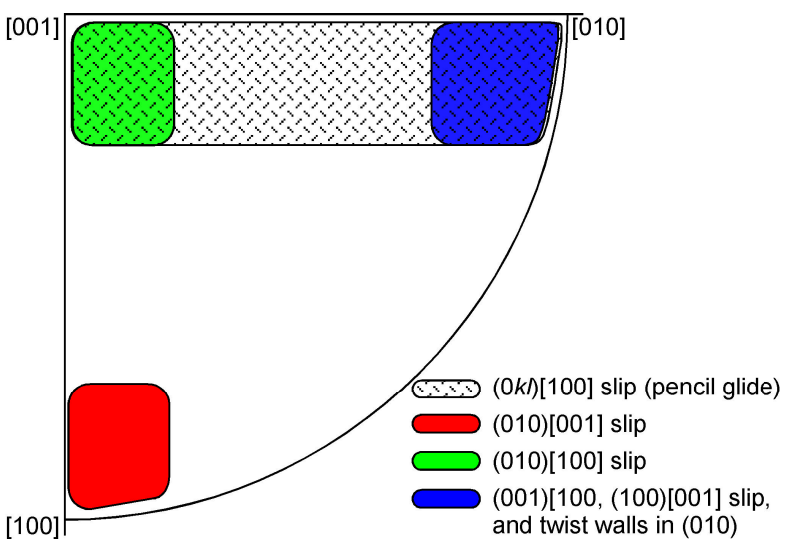

FIG. 2 Orientation of expected sub-grain rotation axes for possible dislocation slip systems in olivine

Fig 3 OIM image quality map and corresponding inverse pole figure map showing the sub-grain structure in an olivine crystal. Yellow lines are boundaries with misorientations $<5^{\circ}$.
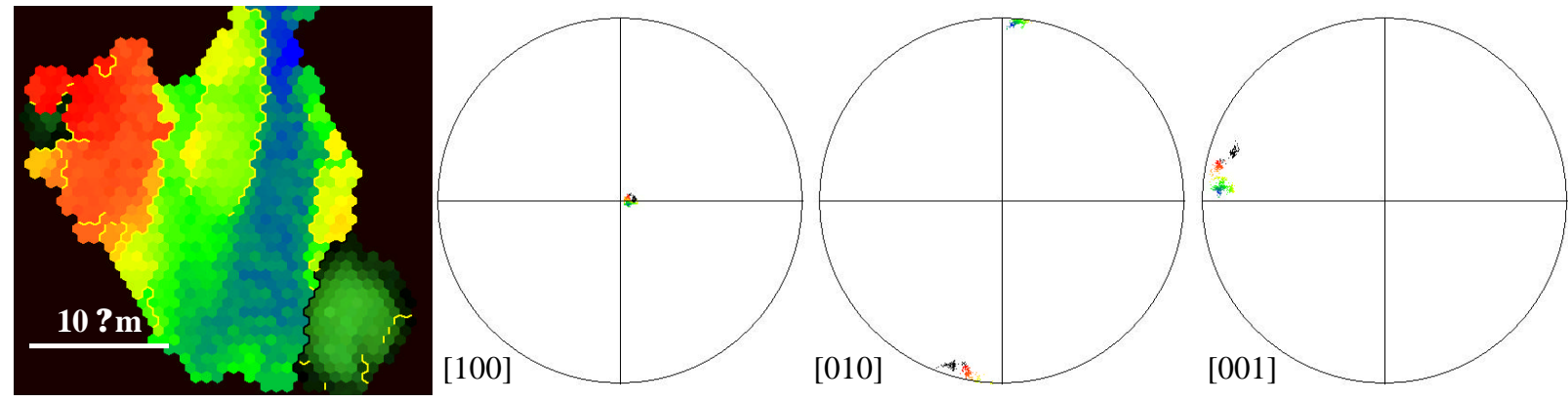

Fig.4 OIM crystal orientation map of an olivine grain with corresponding pole figures in the same colour scheme. The [100] axes are stationary for the whole grain while the [010] and [001] axes progressively rotate, indicating deformation by (010)[001] slip. 